\section{Clindamycin-induced esophageal ulceration}

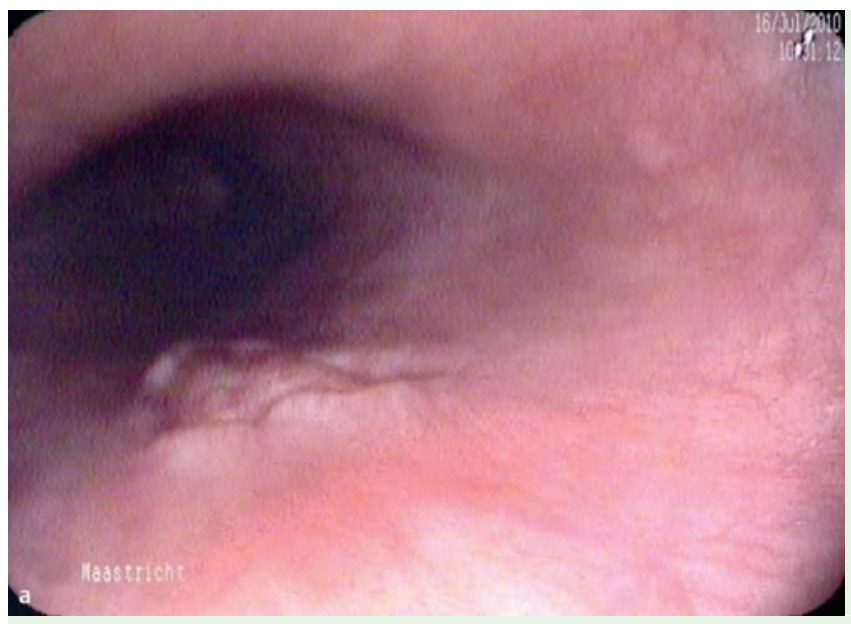

Fig. 1 a, b Endoscopic view of the ulcerative esophageal lesion.

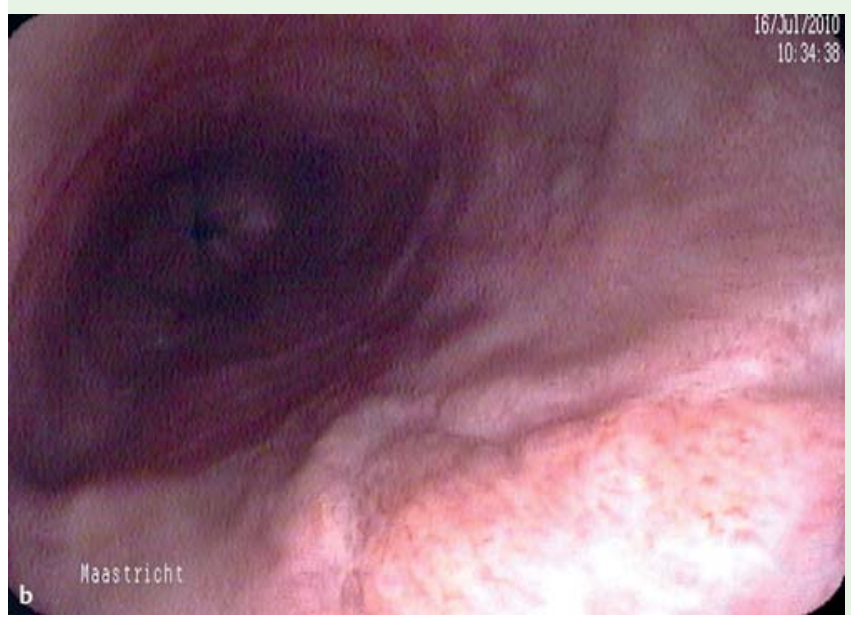

A 24-year-old man, with no prior medical history, presented with acute-onset midchest pain, aggravated by swallowing. The pain had begun 6 days previously when the man was lying down. The patient had taken the last dose of three 300-mg clindamycin tablets for mild folliculitis, 1 hour before onset of the symptoms. Self-prescribed pantoprazole - $20 \mathrm{mg}$ for 3 days had provided no relief.

Physical examination revealed no abnormalities. Upper gastrointestinal endoscopy showed an isolated ulcerative esophageal lesion of $1.5-2 \mathrm{~cm}$ that was $35 \mathrm{~cm}$ from the incisor teeth ( Fig. 1), without signs of erosive reflux esophagitis, and a normal appearance of the stomach and duodenum. Histopathological investigation of the ulcer revealed signs of severe chronic inflammation but no signs of malignancy, cytomegalovirus, Herpes simplex virus, tuberculosis, or yeast infection. The patient's complaints diminished avoided before going to bed.
Although upper gastrointestinal symptoms associated with clindamycin use are uncommon (or underreported), clindamycin-induced ulcers have been reported since 1977 [1,2]. Most clindamycin-induced lesions are self-limiting, and discontinuation of the causative drug is the best treatment [3]. Antacids, proton pump inhibitors, and sucralfate have been used as treatment, although evidence is lacking [3]. In conclusion, clindamycin-induced esophageal ulcerations are rare, but physicians should be aware of this potential adverse effect of clindamycin.

Endoscopy_UCTN_Code_CCL_1AB_2AC_3AZ

\section{Competing interests: None}

\section{G. H. J. Wagenvoort ${ }^{1}$, W. N. K. A. van Mook $^{2}$, A. G. L. Bodelier ${ }^{3}$}

${ }^{1}$ Department of Intensive Care Medicine, Maastricht University Medical Center, Maastricht, The Netherlands

2 Departments of Intensive Care Medicine and Internal Medicine, Maastricht University Medical Center, Maastricht, The Netherlands

3 Department of Gastroenterology, Amphia Hospital, Breda, The Netherlands

\section{References}

1 Sutton DR, Gosnold JK. Oesophageal ulceration due to clindamycin. Br Med J 1977; 1 : 1598

2 Delpre G, Kadish U, Stahl B. Induction of esophageal injuries by doxycycline and other pills. A frequent but preventable occurrence. Dig Dis Sci 1989; 34: 797-800

3 Rivera Vaquerizo PA, Santisteban Lopez Y, Blasco Colmenarejo $M$ et al. Clindamycin-induced esophageal ulcer. Rev Esp Enferm Dig 2004; 96: 143 - 145

The diagnosis of a clindamycin-induced esophageal ulcer was suspected. It is known that delayed passage of capsules or tablets and subsequently prolonged mucosal contact may cause esophageal irritation and ulceration, especially in areas adjacent to the arch of the aorta and left main bronchus. Likewise, this complication has previously been reported after ingestion of other drugs such as aspirin, tetracycline, doxycycline, and potassium chloride. Heartburn, mid-chest pain, and dysphagia are the most frequently reported symptoms. Using small amounts of water and adopting a supine position after ingestion are associated with increased risk of developing esophageal ulceration, and therefore use of these drugs should be

\section{DOI}

Endoscopy 2011; 43: E383 ISSN 0013-726X

\section{Corresponding author}

\section{G. H. J. Wagenvoort, MD}

Department of Intensive Care Medicine

Maastricht University Medical Center

PO Box 5800

P Debyelaan 25

6202 AZ Maastricht

The Netherlands

Tel.: +31-43-3876543

wagenvoort@gmail.com (c) Georg Thieme Verlag KG Stuttgart · New York 